Review Article

\title{
Vaccination programme in India- the present status: a review
}

\author{
Gopalakrishnan S.*, Sujitha P.
}

\begin{abstract}
Department of Community Medicine, Sree Balaji Medical College and Hospital, Bharath Institute of Higher Education
\end{abstract} and Research, Chrompet, Chennai, Tamil Nadu, India

Received: 11 July 2020

Accepted: 13 August 2020

\section{*Correspondence:}

Dr. Gopalakrishnan S.,

E-mail: drsgopal@gmail.com

Copyright: (C) the author(s), publisher and licensee Medip Academy. This is an open-access article distributed under the terms of the Creative Commons Attribution Non-Commercial License, which permits unrestricted non-commercial use, distribution, and reproduction in any medium, provided the original work is properly cited.

\begin{abstract}
Vaccines are substances that are used to provide immunity by stimulating the production of antibodies. Immunization is a process by which a person is made to develop resistance towards infectious diseases by using a vaccine. Immunization programme is one of the key interventions for protection of children from life threatening conditions, which are preventable. By this cost effective intervention program, morbidity and mortality due to vaccine preventable diseases has been drastically reduced. In India, immunization programme was introduced in 1978 as 'Expanded Programme of Immunization' (EPI) by the Ministry of Health and Family Welfare. In 1985 the programme was modified as 'Universal Immunization Programme' (UIP) implemented in a phased manner. Through this programme nearly 12 vaccines are provided to infants, children and pregnant women. Even though the UIP is expected to cover all the eligible children and pregnant women in the country, the overall vaccination coverage falls short of the expected targets in different geographical regions of the country. As per NFHS 4 data, Punjab had the highest and Nagaland had the lowest vaccination coverage and there is a huge gap between the urban and rural areas in India. Through this review article the authors are trying to trace out the history and evolution of the vaccination programmes and the present status of the programme in view of the various difficulties it faces in recent times, which can lead to outbreaks and re-emergence of already controlled vaccine preventable diseases.
\end{abstract}

Keywords: Diseases, EPI, Immunization, Infection, UIP

\section{INTRODUCTION}

\section{Infectious diseases}

Infectious diseases are disorders caused by microorganisms such as bacteria, viruses, fungi or parasites. Many of these organisms live in and on our bodies and are normally harmless or even helpful. But some virulent organisms from outside the body may cause infectious disease.

Infectious diseases are usually transmitted by any of these routes: 1) Direct contact; 2) Indirect contact; 3) Insect bites; and 4) Food contamination.
Some of the infectious diseases can be passed from person to person while insects or other animals transmit some. Consuming contaminated food or water or being exposed to organisms in the environment also transmits infections. Giving appropriate vaccines can prevent many of the dreaded infectious diseases, such as measles, rubella and chickenpox. ${ }^{1}$

\section{Emerging and re-emerging infectious diseases}

Because of the advancement of Medical Sciences, people believed that the age-old battle of humans against infectious disease was virtually over, with humankind the winners, but the events of the past 2-3 decades have 
shown otherwise. Globally, infectious diseases have been remaining the leading cause of death, and they are the third leading cause of death worldwide. Clearly, the battle has not been won, because of the emergence of certain new diseases and re-emergence of certain infections, which we thought, had been controlled. ${ }^{2}$

Emerging infectious diseases are diseases that have not occurred or not recognized in humans before. Examples: AIDS, Ebola hemorrhagic fever, Lyme disease, gastric ulcers etc. Re-emerging infectious diseases are diseases that once were major health problems globally or in a particular country, and then declined dramatically, but are again becoming health problems for a significant proportion of the population. Examples: malaria, tuberculosis, diphtheria, measles, pertussis etc. ${ }^{2}$

Throughout the course of history, disease outbreaks have ravaged humanity, sometimes changing the course of history and, at times, signaling the end of entire civilizations. The worst epidemics and pandemics, which had devastated humanity dating from prehistoric to modern times, were the following: Plague, Yellow fever, Flu pandemic, Polio epidemic, Spanish Flu, Asian Avian Flu, AIDS pandemic and epidemic, H1N1 Swine Flu pandemic, West African Ebola epidemic and Zika virus epidemic.

Recently the COVID 19 Pandemic which is doing havoc around the world also joined the list of the worst pandemics. Now developing countries like India is facing a double edged sword in the form of disease burden due to emerging and re-emerging infectious diseases along with the burden of non communicable diseases as well.

In this context, the development of vaccines to prevent certain life threatening infectious diseases was a boon to the fight against them by reducing the morbidity and mortality due to the infectious diseases. Thus vaccination or immunization became a modern day cost effective scientific advancement in the medical field, in reducing and preventing death and disabilities due to certain life threatening infectious diseases, which have been a major challenge to the medical and public health fraternity

\section{Vaccine preventable diseases}

A vaccine preventable disease is an infectious disease for which there is an effective preventive vaccine exists. With $100 \%$ immunization, and $100 \%$ efficacy of the vaccines, one out of seven deaths among young children could be prevented, mostly in developing countries, The WHO lists 27 diseases for which vaccines are available and vaccine trials are going on for developing vaccines against another 20 infectious diseases. ${ }^{3}$

In spite of the success stories and achievements through the existing immunization programmes and the best efforts taken by each nation worldwide in achieving maximum immunisation coverage, there seems to be a laxity and hesitancy among the people in immunising children and vulnerable people in the society.

Through this review article the authors are trying to trace out the history and evolution of the vaccines and vaccination programmes, the present status of the programme in India in view of the various difficulties and the resistance it faces in recent times, which can lead to outbreaks and re-emergence of already controlled vaccine preventable diseases.

\section{HISTORY AND EVOLUTION OF VACCINATION}

\section{Vaccines}

A vaccine is defined as "a substance used to stimulate the production of antibodies and provide immunity against one or several diseases, prepared from the causative agent of a disease, its products, or a synthetic substitute, treated to act as an antigen without inducing the disease" (Oxford Dictionary). A vaccine helps the body's immune system to recognize and fight pathogens like viruses or bacteria, which then keeps us safe from the diseases they cause. ${ }^{4}$

Vaccines protect against more than 25 debilitating or lifethreatening diseases, including measles, polio, tetanus, diphtheria, meningitis, influenza, typhoid and cervical cancer. Currently majority of the children receive their vaccines on specified time. However, nearly 20 million worldwide still miss-out putting them at risk of serious diseases, death, disability and ill health. ${ }^{4}$

\section{Vaccination}

Vaccination is one of the most cost-effective ways to prevent infectious diseases. Vaccination, or immunization, work by stimulating the immune system which is the natural disease-fighting system of the body. The healthy immune system is able to recognize invading bacteria and viruses and produce antibodies to destroy or disable them to ward off a disease. In addition to the initial immunization process, periodic repeat doses or "boosters" can improve the effectiveness of immunizations. ${ }^{5}$

\section{Vaccine types}

Vaccines are of different types and each type is designed to teach the body's immune system how to fight off certain kinds of germs- and the serious diseases they cause. There are 4 main types of vaccines: ${ }^{6}$

Live-attenuated vaccines: Live vaccines use a weakened (or attenuated) form of the organism that causes a disease. Live vaccines are used to protect against Polio, Measles, Mumps, Rubella (MMR combined vaccine), Rotavirus, Chickenpox and Yellow fever.

Inactivated vaccines: Inactivated vaccines use the killed version of the organism that causes a disease. Inactivated 
vaccines are used to protect against Hepatitis A, Seasonal Flu, Polio and Rabies.

Subunit, recombinant, polysaccharide, and conjugate vaccines: These vaccines use specific pieces of the organism- like its protein, sugar, or capsid (a casing around the germ). These vaccines are used to protect against: HIb disease, Hepatitis B, HPV (Human papilloma virus), Whooping cough, Pneumococcal disease, Meningococcal disease and Shingles.

Toxoid vaccines: Toxoid vaccines use a toxin (harmful product) made by the organism that causes a disease. Toxoid vaccines are used to protect against Diphtheria and Tetanus.

\section{Primary health care and primary prevention}

The concept of primary health care came into limelight in 1978 following the International Conference in AlmaAta, USSR. One of the main component of the services provided as part of primary health care is to provide immunization services to the needy to prevent vaccine preventable disease as per the National Immunization Schedule.

Primary level of prevention is one of the most important components of the country's primary health care services. Health promotion and specific protection are the two interventions practiced as part of primary prevention. Vaccines and immunization of infants and the vulnerable group plays a major role in implementing specific protection measures in the community. Universal coverage of immunization has helped to reduce the morbidity and mortality among children and other vulnerable groups against vaccine preventable diseases throughout the world through the primary health care services. ${ }^{7}$

\section{UNIVERSAL IMMUNIZATION PROGRAMME}

Immunization programme aims to reduce mortality and morbidity due to vaccine preventable diseases (VPDs). Ever since the invention of the first vaccine against smallpox by Edward Jenner in 1796, vaccination has become indispensable healthcare intervention and has saved millions of lives. Immunization program in India was introduced as Expanded Program of Immunization (EPI) in 1978 by the Ministry of Health and Family Welfare, which later became the Universal Immunization Program (UIP) by 1985 . Universal Immunization Programme has always been an integral part of National Health Mission since 2005. The reproductive, maternal, newborn, child and adolescent health $(\mathrm{RMCH}+\mathrm{A})$ strategy which was launched in 2013 , seeks to provide 'continuum of care' with an integrated set of interventions by a three-tiered health system involving immunization as a key element. ${ }^{8}$

Universal Immunization Programme (UIP) is one of the largest public health programmes targeting close of 2.67 crore newborns and 2.9 crore pregnant women annually. It is one of the most cost-effective public health interventions and largely responsible for reduction of vaccine preventable under-5 mortality rate. Under UIP, immunization is providing free of cost against 12 vaccine preventable diseases: A child is said to be fully immunized if child receives all due vaccines as per national immunization schedule within first year age of child. The two major milestones of UIP have been the elimination of polio in 2014 and maternal and neonatal tetanus elimination in $2015 .^{9}$

Tamil Nadu State in India started the immunization programme against six vaccine preventable diseases in 1978. In order to strengthen the programme further, Universal Immunization Programme was launched in 1985. Annually, around 12 lakh pregnant women and 11 lakh infants are being covered under this programme. All pregnant women and their newborns need to be protected against vaccine preventable diseases by using appropriate vaccines to protect them from diseases like TB, Diphtheria, Pertussis, Hepatitis-B, Haemophilus influenza $B$, Tetanus, Polio, Measles, Rubella and Japanese Encephalitis (Table 1 and 2). ${ }^{10}$

Penta-valent vaccine was introduced in Tamil Nadu State from December 2011 onwards. Penta-valent vaccine gives protection against five vaccine preventable diseases namely diphtheria, pertussis, tetanus, Hepatitis-B and Haemophilus influenza-B with fewer needle pricks to a child. The latest immunization schedule followed as per the Universal Immunization Programme is as follows: ${ }^{10}$

Table 1: Immunization schedule in Tamil Nadu: primary vaccination. ${ }^{10}$

\begin{tabular}{|lllll|}
\hline \multirow{3}{*}{ Age } & Vaccines & Dose & Route & Site \\
& BCG & $0.1 \mathrm{ml}$ & Intra dermal & Left upper arm \\
\cline { 2 - 5 } & OPV zero dose & 2 drops & Oral & Oral \\
\cline { 2 - 5 } & Hep B birth dose (within 24 hours) & $0.5 \mathrm{ml}$ & Intra muscular & Antero-lateral aspect of the mid thigh \\
\hline \multirow{3}{*}{$\mathbf{6}^{\text {th }}$ week } & Penta-1 & $0.5 \mathrm{ml}$ & Intra muscular & Antero-lateral aspect of the mid thigh \\
& OPV-1 & 2 drops & Oral & Oral \\
\cline { 2 - 5 } & IPV-1 & $0.1 \mathrm{ml}$ & Intra dermal & Right upper arm \\
\cline { 2 - 4 } & Rota-1 & 5 drops & Oral & Oral \\
\hline $\mathbf{1 0}^{\text {th }}$ week & Penta-2 & $0.5 \mathrm{ml}$ & Intra muscular & Antero-lateral aspect of the mid thigh \\
\hline
\end{tabular}




\begin{tabular}{|lllll|}
\hline Age & Vaccines & Dose & Route & Site \\
\hline & OPV-2 & 2 drops & Oral & Oral \\
\cline { 2 - 5 } & Rota-2 & 5 drops & Oral & Oral \\
\hline \multirow{3}{*}{ 14 $^{\text {th }}$ week } & Penta-3 & $0.5 \mathrm{ml}$ & Intra muscular & Antero-lateral aspect of the mid thigh \\
\cline { 2 - 5 } & OPV-3 & 2 drops & Oral & Oral \\
\cline { 2 - 5 } & IPV-2 & $0.1 \mathrm{ml}$ & Intra dermal & Right upper arm \\
\cline { 2 - 5 } & Rota-3 & 5 drops & Oral & Oral \\
\hline $\begin{array}{l}\text { 9 months (after } \\
\text { 270 days) }\end{array}$ & ${\text { MR }{ }^{\text {st }} \text { dose }}^{\text {JE 1 (in selected districts) }}$ & $0.5 \mathrm{ml}$ & Subcutaneous & Right upper arm \\
\hline
\end{tabular}

Table 2: Immunization schedule in Tamil Nadu: booster vaccination. ${ }^{10}$

\begin{tabular}{|lllll|}
\hline \multirow{4}{*}{$\begin{array}{l}\text { 16-24 } \\
\text { months }\end{array}$} & Vaccines & Dose & Route & Site \\
& DPT 1st booster & $0.5 \mathrm{ml}$ & Intra muscular & Antero-lateral aspect of the mid thigh \\
\cline { 2 - 5 } & OPV booster & 2 drops & Oral & Oral \\
\cline { 2 - 5 } & MR 2nd dose & $0.5 \mathrm{ml}$ & Subcutaneous & Right upper arm \\
\cline { 2 - 5 } & JE 2 (in selected districts) & $0.5 \mathrm{ml}$ & Subcutaneous & Left upper arm \\
\hline $\mathbf{5 - 6}$ years & DPT 2nd booster & $0.5 \mathrm{ml}$ & Intra muscular & Upper arm \\
\hline $\mathbf{1 0}^{\text {th }}$ year & Td single dose & $0.5 \mathrm{ml}$ & Intra muscular & Upper arm \\
\hline $\mathbf{1 6}^{\text {th }}$ year & Td single dose & $0.5 \mathrm{ml}$ & Intra muscular & Upper arm \\
\hline \multirow{3}{*}{$\begin{array}{l}\text { Pregnant } \\
\text { mothers }\end{array}$} & Td1 Early in pregnancy & $0.5 \mathrm{ml}$ & Intra muscular & Upper arm \\
\cline { 2 - 5 } & Td2 Four weeks after Td1 & $0.5 \mathrm{ml}$ & Intra muscular & Upper arm \\
\cline { 2 - 5 } & $\begin{array}{l}\text { Td Booster If received 2 Td doses in a } \\
\text { pregnancy within the last 3 years }\end{array}$ & $0.5 \mathrm{ml}$ & Intra muscular & Upper arm \\
\hline
\end{tabular}

\section{Vaccine efficacy and effectiveness}

Vaccine efficacy and effectiveness are measures that compare the rates of disease between vaccinated and unvaccinated people in a community. Efficacy is measured in controlled clinical trials, whereas effectiveness is measured once the vaccine is approved for use in the general population. From these measures it is possible to identify the proportion of vaccinated people one would expect to be protected by the vaccine. ${ }^{11}$

Vaccine efficacy is proportionate reduction in disease attack rate (AR) between the unvaccinated (ARU) and vaccinated (ARV) whereas vaccine effectiveness is expressed as a rate difference, with use of the odds ratio (OR) for developing infection in spite of vaccination. Vaccine effectiveness predicts how a vaccine reduces disease in a population. By this, the net balance of benefits and adverse effects of the program can also be assessed. Vaccine potency (i.e., vaccine efficacy) is proportional to vaccine effectiveness. Vaccine effectiveness can be affected by various reasons like maintaining cold chain, accessibility of health care, affordability of vaccine which indirectly reflects the vaccination status of the target population. ${ }^{12}$

\section{Herd immunity}

Herd immunity is an important mechanism by which the larger community is protected (also called community immunity). Oral polio vaccine and measles vaccine are well known for producing good herd immunity in the community. For some diseases, if enough people are immune then transmission of the disease is reduced or eliminated. This is particularly so for diseases such as rubella and pneumococcal disease. High vaccine coverage must be maintained in order to prevent the disease reentering the population. ${ }^{11}$

\section{Vaccine potency and cold chain system}

All the vaccines are sensitive biological products. Some of the vaccines are sensitive to freezing, while some are sensitive to heat and others to light. Vaccine potency is ability of the vaccine to adequately protect the vaccinated persons. Vaccine potency can diminish when the vaccine is exposed to inappropriate temperatures and once lost, vaccine potency cannot be regained. So in order to maintain the quality, vaccines must be protected from temperature extremes. Vaccine quality and potency is maintained by using a process known as 'cold chain' that meets specific temperature requirements of the vaccines which ranges from 2-8 degree Celsius at the end user level. ${ }^{13}$ The 'cold chain' is a system of storing and transporting vaccines at recommended temperatures from the point of manufacture to the point of use. The role of the cold chain is to maintain the potency of vaccines in order to produce an adequate immune response in the recipient. There is also a concept called 'reverse cold chain', which is a system of storing and transporting samples at recommended temperatures from the point of collection to the laboratory in order to test the potency of the vaccine. ${ }^{14}$ 


\section{Vaccine failure}

Though immunization programs have a remarkable global achievement, vaccines are neither $100 \%$ effective nor efficacious. ${ }^{15}$ Generally two factors are responsible for vaccine failure. It can be either vaccine related or host related. Vaccine related failure may be due to vaccine attenuation, regimen or administration failure whereas conditions like immunity status of the host, age, genes, nutritional status etc. can be reasons for host related vaccine failure. The former implies the inability to respond to primary vaccination, whereas the latter is characterized by a loss of protection after initial effectiveness. ${ }^{16}$

\section{Side effects of vaccination}

Common side effects post vaccination are local reactions like pain, swelling, redness at the site of vaccination which may or may not include systemic reactions like fever, drowsiness, rash irritability etc. Syncope can occur after vaccination or prior to injection due to anxiety. Other specific adverse effects include minimal risk of intussusception following Rota virus vaccine, febrile seizures post MMR vaccination and anaphylactic reactions post DPT vaccination. Most parents these days have not come across children having vaccine preventable diseases which have taken them to an imbalance between vaccine pros and cons. This gap can be filled by physicians and health workers to discuss about the harms and benefits of vaccine to the beneficiaries. ${ }^{17}$

\section{Vaccine refusal}

In recent times, parental refusal of vaccination is a growing concern for the increased occurrence of vaccine preventable diseases in children. The probable reasons that parents refuse, delay, or are hesitant to vaccinate their child vary widely between parents. They can be one among the 4 categories, which are 1) Religious reasons; 2) Personal beliefs or philosophical reasons; 3) Safety concerns; and 4) A desire for more information from healthcare providers. ${ }^{18}$

Thus the stakeholders have to seriously address this vaccine refusal problem by enhancing information, education and communication and behavioural change continuum activities. The American Academy of Family Physicians do not encourage exemption from vaccination. Parents who refuse to vaccinate their children are made to sign a vaccine refusal form. Policy makers in India can also implement such methods into the vaccination program. ${ }^{17}$

\section{Vaccine coverage and coverage evaluation survey}

Vaccine coverage survey is done to monitor the routine vaccination at various levels in order to evaluate the effectiveness, to see the effect of supplementary vaccination programs, to evaluate the vaccine refusal rate and vaccine dropout rate etc. In addition, it also contributes data of vaccination to evaluate impact on disease burden. ${ }^{18}$

The Universal Immunisation Programme envisages full immunisation of all the eligible children and pregnant women in the country. The UIP was started in 1985 with plan of coverage target of all pregnant women and 85 per cent of all infants against six VPDs by March 1990. With effect from 1990-1991, the vaccination programme became universalized in geographical coverage and the target of UIP was increased to cover 100 per cent of the infants. Three decades after the introduction of the immunization programme, it has partially only succeeded in reducing the burden of vaccine preventable diseases. Though reported vaccination coverage is always higher, there is a wide gap in reported and evaluated coverage in India. ${ }^{19}$

Globally the proportion of children vaccinated has remained stagnant over the past few years. By 2018, out of 195 countries nearly 129 countries reached 90\% coverage of DPT3 vaccine. Most children receive lifesaving vaccines which prevents almost 2-3 million vaccine preventable deaths each year. On the other hand 19.4 million children under 1 year have not received the basic vaccines. Global Vaccine Action Plan (GVAP) works towards strengthening of immunization program, improve monitoring and surveillance. ${ }^{8}$

In 2014 India was declared as Polio free. Ten years from 2000 nearly 153 million children were immunized through measles campaign across South East Asian Region. Successive year, 40.7 million children were immunized in India through supplementary immunization activities against measles. With this we are gradually moving towards measles elimination goal soon. ${ }^{20}$

Up to 9 million routine immunization sessions happen in India annually targeting 26 million children served through 27,000 cold chain stores to attain complete coverage. But as per the NFHS 4 Data, the immunization coverage in India was only $62 \%$ leaving a large group of unimmunized children. There is a huge gap of vaccine coverage between the urban and rural areas in India. Children who belong to the rural areas fail to get complete vaccine coverage according to their age. This gap is found to be maximum in states like Kerala, Chhattisgarh and Haryana. ${ }^{21}$

\section{Full immunization}

A child is considered to be full immunized if the child receives all the due vaccines as per the national immunization schedule within the first year age of the child. Under UIP, immunization is providing free of cost against 12 vaccine preventable diseases: ${ }^{7}$

Nationally against 9 diseases- Diphtheria, Pertussis, Tetanus, Polio, Measles, Rubella, Childhood 
Tuberculosis, Hepatitis B and Meningitis and Pneumonia caused by Hemophilus Influenza type B.

Sub-nationally against 3 diseases- Rotavirus diarrhoea, Pneumococcal Pneumonia and Japanese Encephalitis; of which Rotavirus vaccine and Pneumococcal Conjugate vaccine are in process of expansion while $\mathrm{JE}$ vaccine is provided only in endemic districts.

Inactivated polio vaccine (IPV) has been introduced in UIP as part of Global Polio end-game strategy, to mitigate the risk associated with tOPV to bOPV switch. IPV was introduced in November 2015 initially in 6 states, which was later expanded across the country by April 2016.

Full immunization also means that children under 5 years should receive all 9 doses of prescribed vaccines both as primary vaccination and also the required booster doses. As a part of Mission Indradhanush, vaccines against meningitis and hepatitis are also included. In certain states Japanese Encephalitis and Haemophilus influenza vaccines were added. In 2017, Pneumococcal Conjugate Vaccine (PCV) was added to universal immunization schedule against pneumonia. On October 2017, an Intensified Mission Indradhanush (IMI) was launched with a special drive to reach those not covered by routine immunization program focusing on selected districts and cities to achieve $90 \%$ full immunization. ${ }^{22}$

\section{CURRENT IMMUNIZATION STATUS IN INDIA}

According to National Family Health Survey 4, the vaccination coverage percentage in India was noted to be as follows. Punjab had the highest vaccination coverage of $89.1 \%$ which included $88.7 \%$ among urban population and $89.3 \%$ among rural population. On the other hand Nagaland had the poorest immunization coverage of $35.7 \%$ having urban and rural coverage to be $41.6 \%$ and $33.4 \%$ respectively. The better performing states in India included Punjab $(89.1 \%)$, Goa $(88.4 \%)$, west Bengal $(844 \%)$, Sikkim $(83 \%)$ and Kerala $(82.1 \%)$. Tamil Nadu had coverage of $69.7 \%$ with $73.3 \%$ urban coverage and $66.8 \%$ rural coverage. The poor performing states included Nagaland $(35.7 \%)$, Arunachal Pradesh (38.2\%), Assam (47.1\%), Mizoram (50.3\%) and Gujarat (50.4\%). ${ }^{23}$

\section{Reasons for non-vaccination}

India's immunisation programme is one of the largest in the world, vaccinating around 26.7 million infants and 30 million pregnant women. Despite steady progress, an estimated $38 \%$ of children failed to receive all basic vaccines in the first year of life in 2016. The factors limiting vaccination coverage include large mobile and isolated populations that are difficult to reach, low demand from underinformed and misinformed populations who fear side effects and those who are influenced by anti-vaccination messages.
A recent study done by Gurnani and colleagues report based on their analysis from the Intensified Mission Indradhanush strategy in India, shows the major reasons for non-vaccination as shown below.

Table 3: Major reasons for non-vaccination.

\begin{tabular}{|l|c|}
\hline Reasons for non-vaccination & $\%$ \\
\hline Lack of awareness & 45 \\
\hline Apprehension about adverse events & 24 \\
\hline $\begin{array}{l}\text { Vaccine resistance (reluctance to } \\
\text { receive the vaccine for reasons other } \\
\text { than fear of adverse events) }\end{array}$ & 11 \\
\hline Child travelling & 8 \\
\hline Programme related gaps & 4 \\
\hline Others & 9 \\
\hline
\end{tabular}

They found that during the 2015-16 survey the estimated full immunization coverage for children aged 12-23 months to be $62 \%$ for India as a whole. During 2018 after Intensified Mission Indradhanush, the proportion of children with full immunization coverage was estimated to be $69 \% .^{24}$

\section{Health education and awareness creation about vaccination}

The medical fraternity in the hospitals and health care workers play a major role in recommending vaccines to prevent diseases among children. Though there may be apprehensions about the side effects of vaccination, it is the duty of the health care personnel to listen to parents, clear their doubts and explain the benefits of vaccination. Misinformation on television, social media can lead to vaccine hesitancy which mislead the community. Future Vaccination programs should include strategies to improve awareness and remove fears through appropriate information, education, communication (IEC) and behaviour change continuum initiative (BCCI). ${ }^{25}$

In spite of the best effort by the stakeholders, the rise of conflicting information about vaccines and the ease with which misinformation can spread via media channels, provide a confusing context for parents and for the general population. They often seek assistance from health workers, religious leaders, family members, or other trusted sources, many among them may not be completely aware about the risks and benefits of vaccines. In this context, it is not surprising that some caregivers have become "hesitant" about decisions to vaccinate their children. ${ }^{26}$

Gaps in the immunization coverage- why it is widening?

The National Statistical Office (NSO) survey shows 60\% immunization coverage whereas government report says $83 \%$ coverage. Out of the $90 \%$ institutional deliveries in India only $60 \%$ of under 5 children are fully immunized according to latest survey done by NSO in July 2018. 
This highlights the health systems failure to follow up children born in hospitals on basis of immunization later in their life. According to NSO data, in India just $58.4 \%$ among rural, and $61.7 \%$ among urban areas were fully immunized. In states like Bihar and Uttar Pradesh, were highest number of children are being born, the full immunization coverage is $48 \%$ and $55 \%$ respectively. However, high performing states like Andhra Pradesh and Kerala show an average full immunization of $74 \%$ and $73 \%$ respectively. ${ }^{22}$

\section{CONCLUSION}

Vaccination or immunization is a modern day scientific advancement in the process of reducing and preventing morbidities and mortalities due to certain infectious diseases, which have been a major challenge to the medical fraternity. Vaccines have been developed against about 27 commonly occurring infectious diseases, which helped to control, eliminate and eradicate dreaded diseases. Governments the World over have devised National Immunization schedules and programmes, which are appropriate to their people, based on the recommendations of International Health Agencies like WHO and UNICEF.

India's Universal Immunization Programme is also envisaged to provide immunization free of cost against 12 vaccine preventable diseases through the public health services in the country. The private sector also plays a major role in immunizing a sizeable proportion of the eligible population. After three decades of the start of the country's immunization programmes, the target of achieving universal coverage $(100 \%)$ of the eligible under five children and pregnant women is still a long way to go. The Intensified Mission Indradhanush (IMI) Programme is expected to bridge this gap and will help to achieve universal vaccination coverage. After the eradication of smallpox in 1977, the two major milestones of UIP have been the eradication of poliomyelitis in 2014 and maternal and neonatal tetanus elimination in 2015.

Funding: No funding sources

Conflict of interest: None declared

Ethical approval: Not required

\section{REFERENCES}

1. Mayo Clinic. Infectious diseases- Symptoms and causes. Available from: https://www.mayoclinic. org/diseases-conditions/infectious-

diseases/symptoms-causes/syc-20351173. Accessed on 8 April 2020.

2. National Institutes of Health. Understanding emerging and re-emerging infectious diseases.. Available from: https://www.ncbi.nlm.nih.gov/ books/NBK20370/. Accessed on 16 April 2020.

3. World Health Organization. Vaccines and diseases. Available from: https://www.who.int/immunization/ diseases/en/. Accessed on 8 April 2020.
4. World Health Organization. Vaccines and immunization. Available from: http://www.who.int/topics/vaccines/en/. Accessed on 4 April 2020.

5. Shiel WC. Medical definition of vaccination. Medicine Net. Available from: https://www.medicinenet.com/script/main/art.asp?ar ticlekey=5925. Accessed on 4 April 2020.

6. Vaccine. Vaccine Types. Available from: https://www.vaccines.gov/basics/types. Accessed on 4 April 2020.

7. Park K. epidemiology of Communicable Diseases: Rabies. In: Park's textbook of preventive and social medicine. 25th edn. Jabalpur: M/s Banarsidas; 2019.

8. World Health Organisation. Immunization coverage. Available from: https://www.who.int/newsroom/fact-sheets/detail/immunization-coverage. Accessed on 5 April 2020.

9. National Health Mission. Immunization. Available from: https://nhm.gov.in/index1.php?lang=1\&level $=2 \&$ sublinkid $=824 \&$ lid $=220$. Accessed on 4 April 2020.

10. Directorate of Public Health and Preventive Medicine. Immunization. Available from: http://www.tnhealth.org/dph/dphis.php. Accessed on 13 February 2020.

11. Immunisation Advisory Centre. Efficacy and effectiveness. Available from: https://www.immune.org.nz/vaccines/efficiencyeffectiveness. Accessed on 4 April 2020.

12. Weinberg GA, Szilagyi PG. Vaccine epidemiology: efficacy, effectiveness, and the translational research roadmap. J Infect Dis. 2010;201(11):1607-10.

13. World Health Organisation. The vaccine cold chain. Available

from:https://www.who.int/immunization/documents/ IIP2015_Module2.pdf. Accessed on 4 April 2020.

14. World Health Organization. EPI logistics. Available https://www.who.int/countries/eth/areas/immunizati on/epi_logistics/en/index1.html. Accessed on 4 April 2020.

15. Heininger U, Bachtiar NS, Bahri P, Dana A, Dodoo A, Gidudu J, et al. The concept of vaccination failure. Vaccine. 2012;30(7):1265-8.

16. Wiedermann U, Garner-Spitzer E, Wagner A. Primary vaccine failure to routine vaccines: why and what to do? Hum Vaccine Immunotherap. 2016;12(1):239-43.

17. Spencer JP, Pawlowski RH, Thomas S. Vaccine adverse events: separating myth from reality. Am Fam Phys. 2017;95(12):786-94.

18. McKee C, Bohannon K. Exploring the reasons behind parental refusal of vaccines. J Pediatr Pharmacol Therap. 2016;21(2):104-9.

19. Lahariya C. A brief history of vaccines and vaccination in India. Indian $\mathrm{J}$ Med Res. 2014;139(4):491.

20. World Health Organisation. WHO urges countries to intensify immunization efforts. Available from: https://www.who.int/southeastasia/news/detail/19- 
04-2012-who-urges-countries-to-intensify-

immunization-efforts. Accessed on 5 March 2020.

21. UNICEF. Immunization. Available from: http://unicef.in/whatwedo/3/immunization. Accessed on 5 March 2020.

22. The Times of India. Full Immunization: Government says $83 \%$, NSO survey shows $60 \%$. Available from: https://www.pressreader.com/india/the-times-ofindia-mumbai-edition/20191203/282888027551832. Accessed on 3 December 2019.

23. National Family Health Survey. Vaccination Coverage. Available from: http://rchiips.org/NFHS/factsheet_NFHS-4.shtml. Accessed on 6 April 2020.

24. Gurnani V, Haldar P, Aggarwal MK, Das MK, Chauhan A, Murray J, Arora NK, Jhalani M, Sudan $\mathrm{P}$. Improving vaccination coverage in India: lessons from Intensified Mission Indradhanush, a crosssectoral systems strengthening strategy. BMJ. 2018;363.

25. Times of India- Chennai News. 1 in 20 Chennai parents averse to vaccines. Available from: https://timesofindia.indiatimes.com/city/chennai/1in-20-chennai-parents-averse-tovaccines/articleshow/72235380.cms. Accessed on 3 December 2019.

26. Benjamin H, Sherine G, Rafael O. Vaccine. Vaccine Special Issue on Vaccine Hesitancy. 2015;33(34):4155-6.

Cite this article as: Gopalakrishnan S, Sujitha P.

Vaccination programme in India- the present status: a review. Int J Community Med Public Health 2020;7:3746-53. 